\title{
Integrated Multi-purposed Testbed to Characterize the Performance of Internet Access over Hybrid Fiber Coaxial Access Networks
}

\author{
Hung Nguyen Chan, Belen Carro Martinez, Rafa Mompo Gomez, and \\ Judith Redoli Granados \\ Department of Signal theory and Telematics. \\ Aula Cedetel - University of Valladolid. \\ 47011 Valladolid - Spain. \\ hungnc@gmx.net \\ http://go.to/hungnc
}

\begin{abstract}
This paper presents an experimental testbed to study the noise effect on the performance of the transport layer over Hybrid Fiber Coaxial (HFC) networks. We have successfully designed and implemented an integrated complex testbed, which is suitable not only for laboratory environments but also reusable for real-world networks. The main purpose of the testbed is modeling the residential broadband data network using hardware simulation under several noise conditions and observing the effects on the performance of popular Internet applications as well as native TCP/UDP performances. A large number of public domain Internet measurement tools have been evaluated, from which several selective software tools have been used. In addition, new software has been developed to combine all software and hardware devices. Based on the testbed, we were able to study several issues of TCP/UDP over HFC networks by making a large number of automatic measurements and analysis. The testbed infrastructure would be very useful for cable operator and end users for monitoring and troubleshooting HFC networks, and can be effectively reused for related studies in similar environments such as wireless and DSL.
\end{abstract}

\section{Motivation}

Dramatic growth of the Internet has motivated the booming of broadband access technologies. Among those, Hybrid Fiber Coaxial (HFC) is one of the most popular access technologies, which provides users not only with TV programs but also high-speed Internet access and other applications. Many HFC characteristics affect the performance of Internet applications running over it, such as asymmetry, tree-and-branch topology, interferences on reverse path, etc, which requires significant considerations.

As the performance of Internet application directly reflects the cable user's satisfaction, cable operators have strong motivations to monitor not only the status of 
cable modem but also the Internet application performance of users' hosts in order to effectively tackle their problems. Also, it is desirable to quickly classify and isolate the network problems. In reality, the Internet access speed of cable users depends on many factors including: RF-related factors, network congestion, QoS parameters, the load of Cable Modems Termination Systems (CMTS), etc. As a result, both users and cable operators need software tools for troubleshooting, and obtaining information about network health. This task is rather difficult without the assistance of special Internet measurement software (which most likely runs on UNIX platforms).

Regarding these issues, we have setup a multi-purposed experimental testbed to study the performance of transport layer over HFC networks. The testbed was targeted to be reused in real-world HFC networks. The additional objective of this study is to answer several questions:

[a] The possibilities of locating noise-affected area based on measuring Internet performance of hosts connected to a same CMTS (as a result, are under similar network conditions).

[b] The effect of noise on Internet applications.

[c] How to quickly distinguish between general network congestions problem and HFC network problems?

The rest of this article is organized as follows: Section 2 provides a brief background on HFC networks. Section 3 describes the experimental testbed. In section 4 , we discuss on the related studies and the contributions of this work. Several results and possible applications of the testbed are illustrated in section 5. Finally, our conclusion and future work are given in the last section.

\section{Overview on HFC Networks}

Figure 1 depicts a typical HFC system that provides residential broadband services. In order to bring data to cable user homes, the digital signal is converted into analog signal and mixed with CATV analog signal using frequency multiplexing. The high band 500-800 MHZ is used for downstream data and the low band from 5 to $40 \mathrm{MHZ}$ is used for upstream data from cable modems.

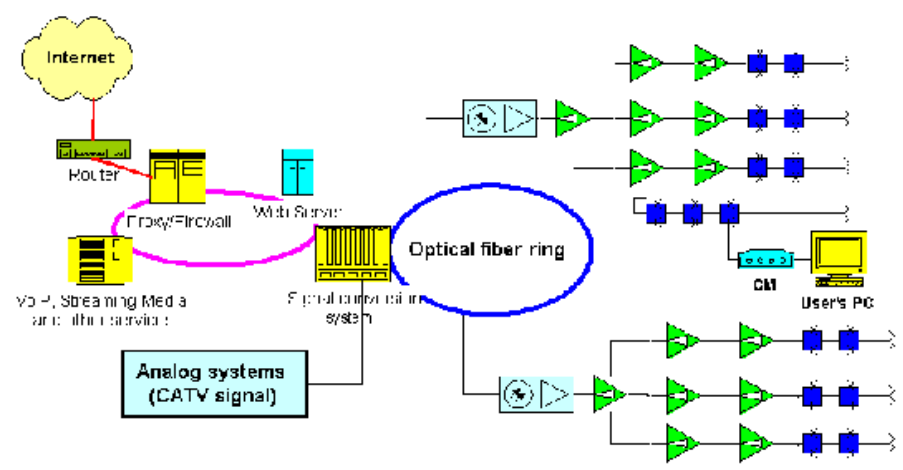

Fig. 1. A typical HFC system providing broadband data services 
HFC networks are highly susceptible to noise funneling, the effect of noise entering the coaxial plant, being amplified through return path amplifiers, and aggregated from other coaxial network branches.

\section{The Experimental Testbed}

\subsection{Hardware Configuration}

Figure 2 illustrates the testbed hardware configuration. We used 6 PCs 1 running multi-operating systems including Linux RedHat 7.1, FreeBSD 4.3, Windows 2000 Server and Windows 98 SE. These OSes can be switched over from a remote PC, which controls the entire testbed. Simulated noise was generated on a PC-controlled arbitrary waveform generator HP33120 and injected into various points of the experimental network. Noise was reproduced using a noise database, taken from operating HFC networks. Another generator (HP3325B) triggered the HP33120 to control the repeated frequency of noise bursts. As a result, all noise parameters such as inter-arrival time, noise form, noise amplitude, etc, of Gaussian and impulse noise, can be fully controlled. An oscilloscope HP 54616, and a CATV analyzer HP Calan 3010R were used to monitor the signal during test. All the equipment and PCs were controlled and monitored from a remote desktop. With an additional PC-control RF switch, the connection configuration can also be changed remotely.

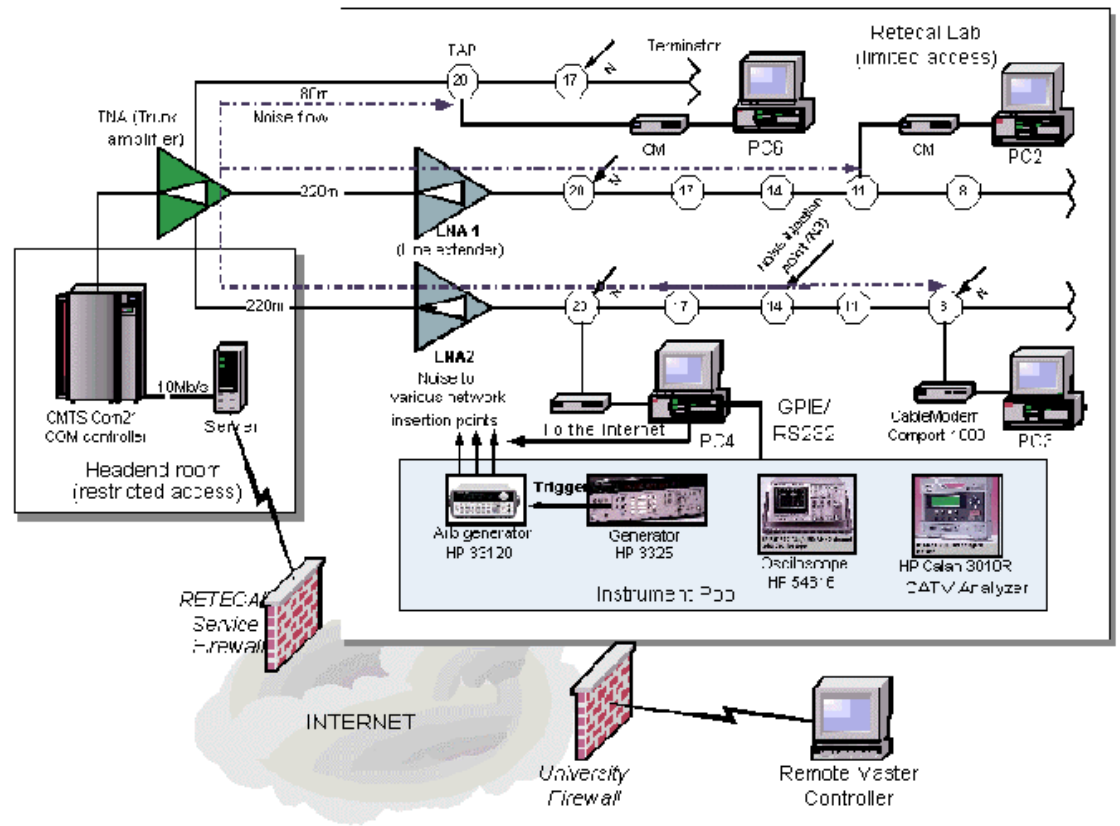

Fig. 2. The testbed hardware configuration

\footnotetext{
${ }^{1}$ Four PCs connected with Cable Modem have similar hardware and software configurations.
} 


\subsection{Software Configuration}

\section{The Overall Software Architecture}

The testbed software structure is depicted in Figure 3. A typical client-server network providing Web and FTP service was simulated, along with a distributed measurement network. A server running Linux RedHat 7.1 provided FTP and Http services for 4 client PCs running Win98SE/LinuxRH. A remote multi-OS PC acted as master controller, controlled the whole testbed through the Internet.

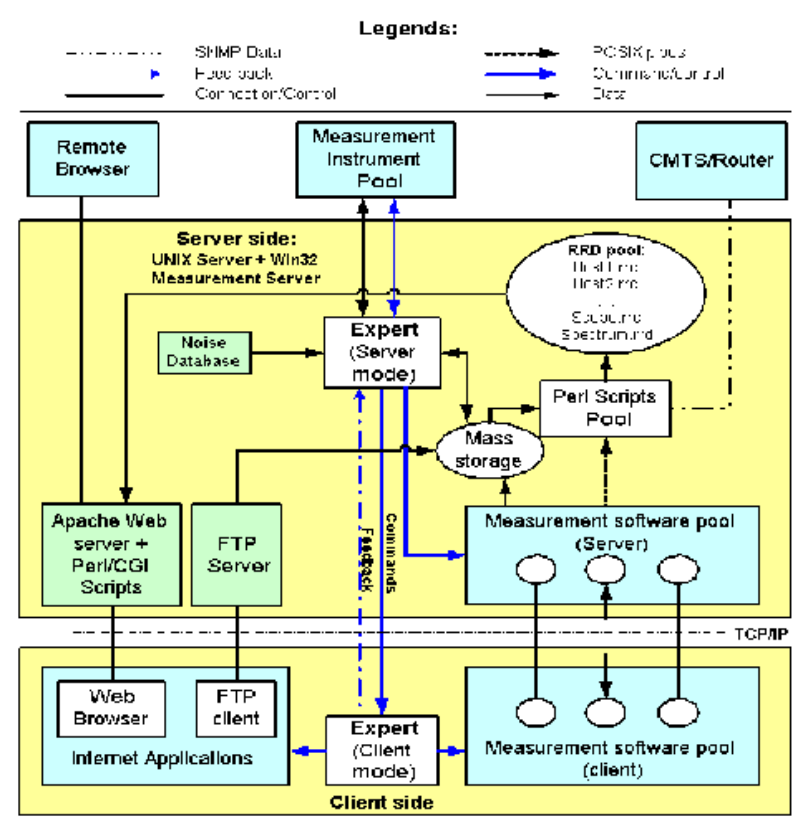

Fig. 3. Testbed software architecture

The software "Expert" plays the main role in the testbed. On the server side, a master "Expert" node can connect to a number of "Expert" slave nodes by either listening to incoming connection or actively connect to listening "Expert" slaves through direct TCP connections or SSH (Secure Socket Host) forwarding. After the communications has been successfully setup, the master node runs a master script in order to control these nodes to launch measurement programs, console commands or Internet applications (e.g. FTP, Web browser) in separated threads?

One "Expert" node, which acted as instrument-control server, handled a number of measurement equipments through GPIB/RS232 interfaces and saves data into a local hard disk for uploading to the Linux server. Data including log files (from servers and clients) and measurement data (from measurement instruments), is processed by various Perl scripts, and then put into a dynamically-created array of Round Robin Databases (RRD). Those scripts can also directly get run-time data from the server-

${ }^{2}$ Slave scripts can also run simultaneously with master script. 
side measurement software pool through POSIX pipes. Another set of Perl/CGI scripts (run on top of Apache Web server) dumps selected data sources from the RRD database pool, make run-time graphs on the requests of remote Web browsers. Optionally, data from CMTS and Routers can also be acquired through SNMP interfaces and put into RRD pool.

\section{The Main Testbed Software: "Expert"}

In order to facilitate the experiment an experimental software, named "Expert", was developed. The primary objective of the software is to provide a communication links for measurement nodes and a simple graphical interface to execute/debug measurement scripts. An integrated measurement script can combine Win32 shell commands, Unix-ported commands, Windows scripting host, scripts written in scripting languages such as Perl/TCL, and a number of additional internal UNIX-style commands and communication commands. A special communication protocol was implemented in the software so that the measurement nodes can work in both clientserver and peer-to-peer architecture. (More details can be found in [15], which was written by the same author.)

Moreover, the event-based feedback and the capability to control measurement instruments of the software would also be useful for the traditional HFC status monitoring on the physical layer. The design of "Expert" software was based on our previous experiences and codes [3], [10] and regarding related software and measurement techniques [4]. The software "Expert" was written using Visual Basic 6 on the client side and Perl 5.6 on the server side 3 .

\section{Internet Measurements}

During the project, a large number of public domain tools have been evaluated on UNIX (Linux and FreeBSD) and Win32. These measurement tools are the result of many studies of the academic and OpenSource community.

Among these tools, the most useful are: 1) Iperf to characterize the native TCP/UDP bandwidth from cable PCs to CMTS 2) NCS to characterize the path from CMTSs toward the Internet backbone 3) A combination of Tcpdump/Windump/ Tcptrace/Xplot to analyze packet-level traces and 4) Ntop, a modern passive measurement software. 5) Traditional ping.

\section{Related Studies and Contributions of This Work}

At present, few studies have focused on the performance of Internet applications and protocols run over HFC since the majority of the studies ([1], [5]) focused on the physical layer in order to find solutions to detect and mitigate cable upstream interferences, which severely degrade digital services. Among these studies, S. Chaterjee [6] and P. Tzerefos [12] using OPNET, a commercial simulation software, R. Cohen [2] used NS2 simulator in order to simulate TCP-based applications over HFC network. However, these studies did not directly regard the effects of

3 The information on the availability of the testbed software can be found on the author 's home page: http://go.to/hungnc. 
interferences on HFC networks. To our knowledge, one of the reasons for this is the complexity of simulating both the digital system providing Internet services and analog systems and signals only by using software simulations. Another reason is the high-cost of HFC network equipment such as CMTS, or broadband router. In addition, the HFC measurements taken on real HFC systems must be non-intrusive so as not to affect the operating network.

In the Internet measurement field, many studies have been successfully performed for decades in both traditional network environments [4], [9] as well as new environments such as wireless [13]. Numerous measurement tools have been introduced as a result of those studies.

Our previous work [3] was primarily concerned with monitoring the HFC physical layer performance by making use of a set of measurement instruments such as spectrum analyzers and oscilloscopes, which is a traditional approach. However, due to additional cost, many cable operators do not perform the preventive maintenance routines, which aim at monitoring the physical layer, even though these detailed routines have been clearly defined for years. In the next phase of our project [10], testbed software "Expert" was developed and provided automatic data collection on Win32 platform. In the current phase, server side software has been significantly improved by cross-platform Perl/CGI programs and Round Robin Database (RRD), which allow real-time display and analysis on popular Apache Web server while the client side has the new feedback capabilities.

One of our greatest challenges was the complexity of the experimental testbed. This involved a large range of issues including; controlling measurement equipment, investigating the characteristics of HFC network and Internet services from the physical layer up to the application layer, synchronizing distributed network measurement, dealing with communication with remote measurement instruments through Internet firewalls, (which eliminated the feasibility of most commercial software products such as the measurement software that implements protocols such as Agilent SICL LAN, TCP/IP VXI and MS DCOM).

In addition, most current Internet measurement tools are primarily available on UNIX platforms while the ATE (Automatic Test and measurement) software is most likely available only on Win32 platform or some proprietary platform (such as HPUX). Our work successfully integrated Internet measurement tools with measurement equipment and software, as well as simulated typical Internet services over HFC networks. The testbed infrastructure allowed large range of studies such as the interactions between the physical layer and the link layer [11], the physical layer and the transport layer [10], and interestingly, an approach to locate noise affected area based on measuring transport layer performance, which will be very cost-effective. Several results and possible applications of the testbed will be presented in the next section.

\section{Results: Possible Applications of the Testbed Infrastructure}

\subsection{Characterizing TCP/IP Performance over HFC under Noise Conditions}

The testbed infrastructure allows studying numerous problems of HFC, such as noise funneling effects on cable user's applications, finding the vulnerable points of the 
networks to focus monitoring and maintenance efforts, detecting malicious-purpose noise injections, as well as the issues mentioned in section 1 . Since most of the tests can be run automatically and unattended, this allows for a large number and variety of tests.

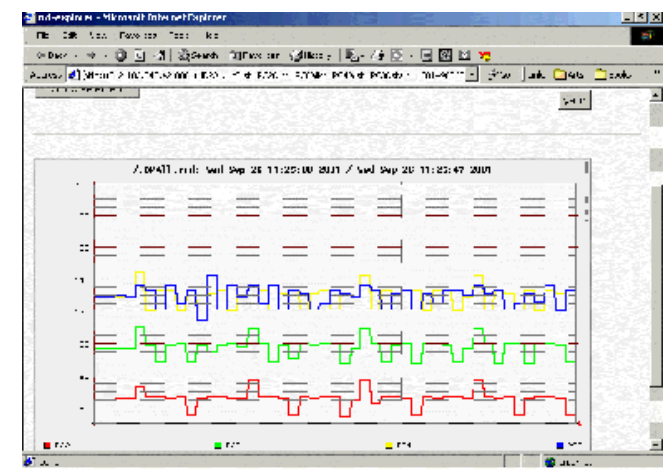

Fig. 4. Upstream UDP throughput under noise (run-time graph)

\section{Locating the Noise Injection Based on the Noise Isolation Factor}

Based on the relative location of computers and the noise injection point shown in Figure 2, the calculation of noise isolation factor is shown in Table 1:

Table 1. Noise isolation factor

\begin{tabular}{|c|c|}
\hline $\mathrm{PC}$ & Noise isolation (I) (for the connection scheme in Figure 2) \\
\hline PC2 & $\begin{array}{l}14(\text { tap })+\text { cable* } 3+(- \text { LNA2 }+220 \mathrm{~m})+220 \mathrm{~m} * 2,5 \mathrm{db} / 100 \mathrm{~m}(\mathrm{at} \\
40 \mathrm{MHZ})+4(\text { TNA combiner loss })+\mathrm{LNA} 1(19)+\text { cable } * 4+11 \\
(\operatorname{tap})=54 \mathrm{~dB}\end{array}$ \\
\hline PC6 & $\begin{array}{l}14(\text { tap })+\text { cable } * 3+(- \text { LNA } 2+220 \mathrm{~m})+4(\text { TNA combiner loss })+ \\
80 \mathrm{~m} * 2,5 \mathrm{db} / 100 \mathrm{~m}+20(\text { tap })=40 \mathrm{~dB}\end{array}$ \\
\hline PC3 & $14(\operatorname{tap})+$ cable $* 2+8(\operatorname{tap})=22 \mathrm{~dB}$ \\
\hline PC4 & $14(\operatorname{tap})+$ cable $* 2+20(\operatorname{tap})=34 \mathrm{~dB}$ \\
\hline
\end{tabular}

While observing the table, it should be noted that the losses of short cable between taps are assumed to be zero, and $(-\mathrm{LNA} 2+220 \mathrm{~m})=0 \mathrm{~dB}$ since the testbed coaxial network was calibrated for unity gain, which means that the gain of an amplifier equals the losses that precede it. (See [7])

Figure 4 is a screenshot of a run-time graph displaying the UDP measurement results using Iperf 1.2, (with the bandwidth parameters set in accordance with the QoS parameters assigned for CMs) on 4 clients. In this figure, the noise effects on cable users can be observed. The left-most flat section corresponds with the normal operation before various impulse noise forms of increasing amplitude were injected into the network. The noise injected in point N3 (See Figure 2) causes UDP bandwidth fluctuations. It can be observed that the effects on 4 PCs are very different: PC 3 seemed not be affected until noise exceeds a threshold. The main reason is the differences of the noise isolation factors relative to the noise injection point. 
We also realized that among various parameters such as round trip delay, jitter, etc, the fluctuation of UDP bandwidth is a good metric to assess the noise isolation factor, in order to predict the noise injection location.

$$
\begin{aligned}
& \sigma=\sqrt{\frac{1}{n} \sum_{1}^{n}\left(x_{i}-\bar{x}\right)^{2}} \\
& \rho_{X Y}=\frac{\operatorname{cov}(X, Y)}{\sigma_{X} \sigma_{Y}}
\end{aligned}
$$

With standard deviation and correlation calculated using equation (1) and (2), respectively, the correlation between the standard deviation of UDP throughput (which represents for the UDP bandwidth fluctuation) and the isolation factor (calculated in Table 1) is -0.90338 , and the correlation strength is 0.816097 , which is satisfactory.

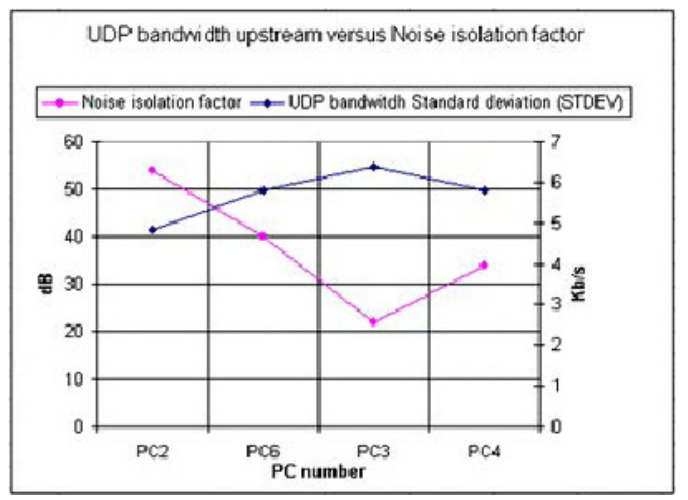

Fig. 5. Standard deviation of native UDP bandwidth versus noise isolation factor

The importance of this finding is as follows:

- Based on the estimation of noise isolation factor, together with experiences on operating networks, a vulnerable map of the network can be established (See [15]).

- If the fluctuations of UDP bandwidth are detected on a number of cable users or dedicated measurement nodes, it is most likely that noise has affected the areas that have low noise isolation relative to these measurement nodes. Typically, these areas are neighborhood taps and the taps near the bi-directional amplifiers, where upstream noise is amplified ([15]). This fact can assist in locating noise injection point, which is the solution for issue [a] mentioned in section 1 .

Table 2. The correlations between noise isolation factor and various performance metrics

\begin{tabular}{lcc}
\hline \multicolumn{1}{c}{ Performance metrics } & Correlation Coefficient & Correlation Strength \\
\hline Native UDP upstream & $-0,903$ & 0,816 \\
Native TCP upstream & -0.845 & 0.714 \\
(measured by Iperf) & & \\
FTP bandwidth upstream & -0.807 & 0.615 \\
FTP bandwidth downstream & -0.796 & 0.633 \\
\hline
\end{tabular}


As can be seen in table 2, the native UDP bandwidth, native TCP bandwidth, FTP bandwidth upstream and FTP bandwidth downstream are in descending order of correlation strength. This is consistent with our expectation.

\section{Characterizing the Effects of Noise on Internet Applications}

In order to investigate issue [b] in section 1, two typical Internet applications, FTP and WWW (using MSIE 5.5) were characterized in the testbed. Several results are shown on Figure 6.
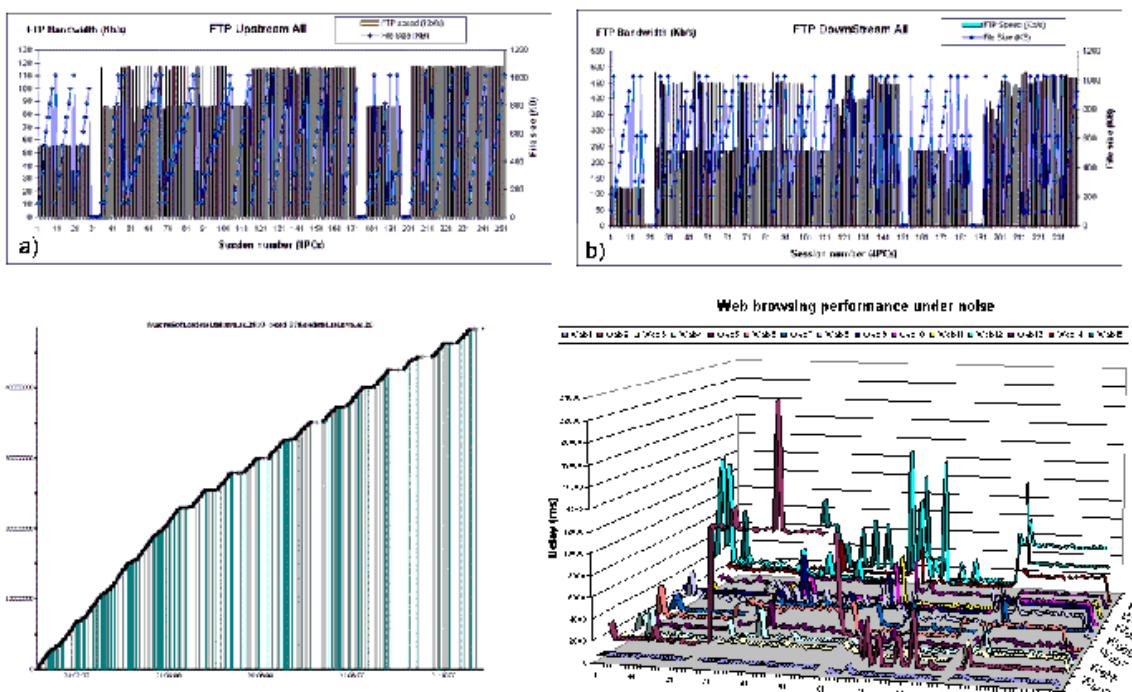

c)

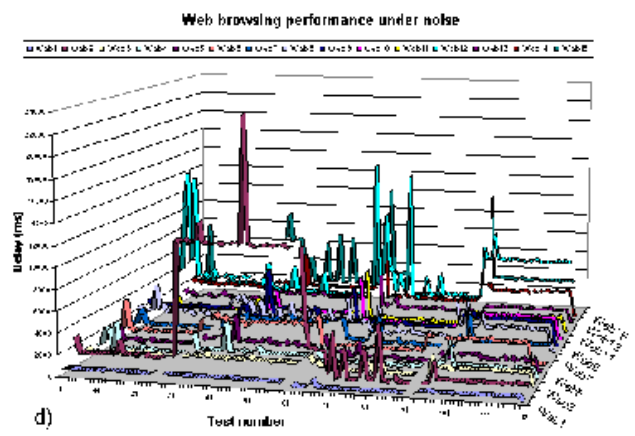

Fig. 6. Characterizing Internet applications a) FTP upstream b) FTP downstream c) A packetlevel trace shows retransmissions of a FTP upstream session due to impulse noise d) Excessive Http delay and timeout due impulse noises.

As can be seen on Figure 6, under similar noise condition, the effect of ACK loss on FTP downstream throughput (Fig. 6b), can degrade throughput up to $50 \%$, while the effect of packet loss on FTP upstream, only degrade throughput up to $25 \%$ (Fig. 6a). Web browsing is more susceptible to noise effects than FTP 4 (Figure 6d). In addition, the effects on Web browsing also heavily depend on Web page size and structure.

4 However, in reality, users normally do not wait until a Web page is fully loaded before surfing to another page. 


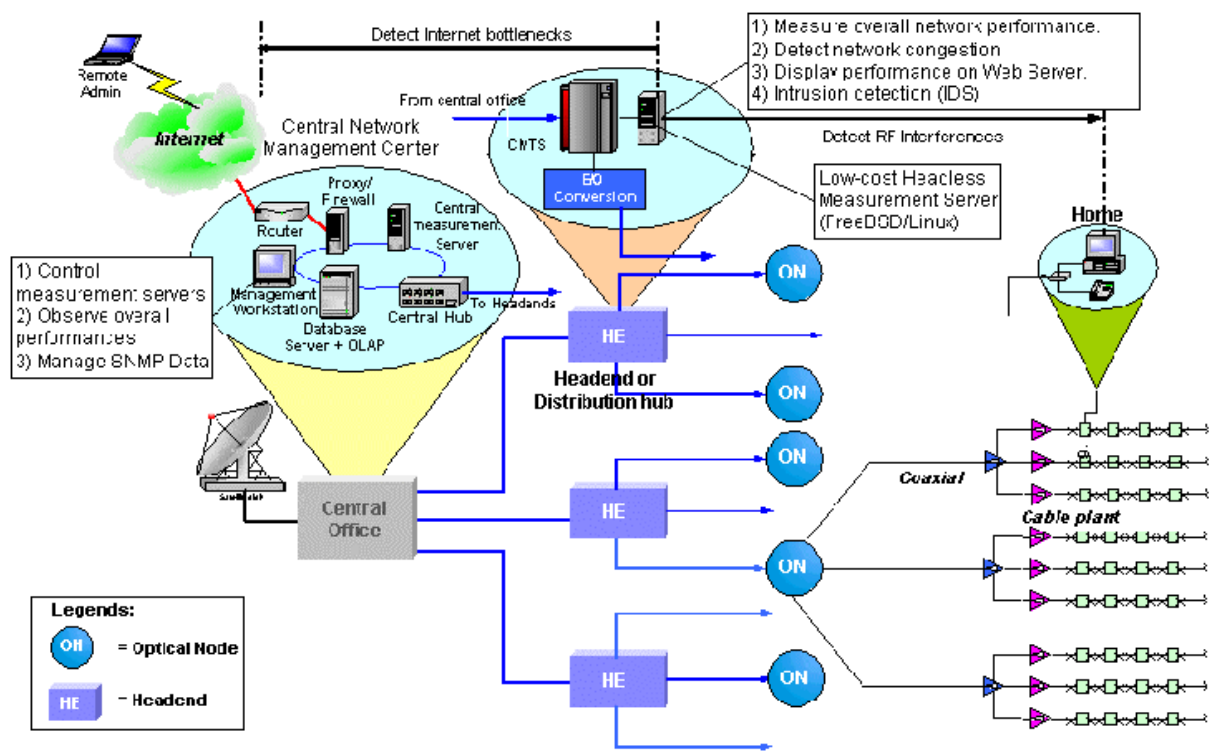

Fig. 7. Measurement network on HFC infrastructure

\subsection{Other Applications}

\section{Monitoring HFC Network}

On the next phase of the project, we are planning a measurement infrastructure based on the current testbed. Figure 6 shows the suggested measurement network, which is based on the current testbed. The measurement network will contain low-cost headless servers running FreeBSD or Linux, which are located at the network distribution centers to serve 500 to 2000 cable users. The main measurement toolset will be installed at the server while end-user software can be downloaded freely to help users monitoring and troubleshooting network health. The servers can characterize the path toward Internet backbone to detect network bottleneck as well as the path toward cable users to detect RF-related problems. Therefore, they are able to solve the issue [c] previously mentioned in section 1 . In this scenario, if a software is installed on the user side, it can perform some types of measurement and diagnosis and help users to tune their network setting to obtain higher performance in HFC environments, while the measurement results can also be seen on the servers due to the client-server nature of several Internet measurement software (e.g. Iperf). In addition, user-side software can greatly assist in topology discovery, trouble-shooting and improve the precision of Internet measurement.

\section{Benchmarking Cable/DSL Modems and CMTS/DSLAM}

As described in [8], measurement procedures to benchmark Cable/DSL modems and/or CMTS/DSLAM can be performed by a commercial system such as Smartbit. However, in the absence of such a high-cost system, a modified testbed infrastructure 
can be used. Figure 7 illustrates the implementation of the testbed infrastructure as a benchmark system.
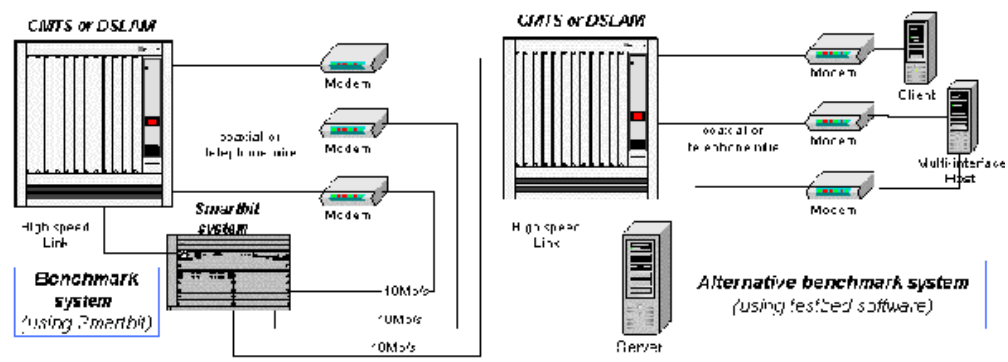

Fig. 8. Using Testbed infrastructure as a benchmark system

\section{Other Access Network Environments (DSL, Wireless, etc)}

It is widely known that DSL networks suffer from similar problems as HFC network such as noise and interferences. Moreover, since telephone wire is more susceptible to noise in comparison with coaxial, DSL performance is dramatically degraded with the presence of noise. In the very designing phase, the testbed was aimed to be transparently reused in DSL environments with very small or no modifications.

\section{Conclusions and Future Work}

This paper has described an experimental integrated testbed to study the overall performance of Internet access over HFC networks under simulated noise conditions and discussed on the applications of the testbed infrastructure. From the testbed experiences, a large number of measurement tools were collected and evaluated on the HFC infrastructure. The testbed infrastructure is flexible enough to be easily adapted for other purposes as well as other network environments.

One of the interesting results obtained from the experimental testbed is the novel approach of locating noise injection points based on the correlation between the noise isolation factor and the variation of UDP bandwidth. The implementation of this approach in real networks would require some network topology discovery techniques. For this purpose, there are several possibilities: 1) Obtaining ranging parameters of CMs through SNMP interfaces of CMTS. 2) Cable users can provide their CM locations themselves through client software or a database-backend Web page. 3) The network maps are already available by network designing and maintenance procedures. 4) A combinations of the above three solutions. However, the testbed still have several limitations, such as the scale of the testbed was small in comparison with real HFC networks, etc. We will address those limitations in the next phase of the project by making non intrusive-measurements on the real operating HFC networks. The server-side software will be able to assist the automatic analysis more effectively if its database capability is improved. One possibility is connecting the current RRD database pool with an OLAP engine (online analytical processing), to form multi-dimensional databases of HFC network performance. 
Acknowledgements. This work is supported by Cedetel, Spain, and Retecal, a Spanish cable operator. The author also appreciates the anonymous reviewers for their comments on the previous version of this paper and the OpenSource community, who made many valuable software and source code available.

\section{References}

1. K.Hui Li, "Impulse noise identification for the HFC upstream Channel" IEEE transaction on broadcasting, vol. 44. No.3, pp 324-329, September 1998.

2. R. Cohen, S. Ramanathan, "TCP for high performance in hybrid fiber coaxial broadbandaccess networks", IEEE/ACM Transaction on networking, pp 15-29, vol. 6. No.1, February 1998.

3. N. Chan Hung, R. Mompo, J. Redoli, B. Carro, "Flexible COM-based software solution for HFC network monitoring”, Proceeding of IFIP TC6/WG6.7, Smartnet 2000, pp 555568, Kluwer Academic Publisher. Available: http://www.portalvn.com/hungnc/CVRevised.htm

4. CAIDA Internet measurement taxonomy. Available on http://www.caida.org

5. C. A. Eldering, N. Himayat, F. M. Gardner, "CATV Return path characterization for reliable communications", IEEE Communication magazine, Aug 1995, pp 62-69.

6. S. Chatterjee, L. Jin , "Performance of residential broadband services over High-speed cable networks", Proceeding of Workshop on Information Technology and Systems (WITS98), Helsinki, Finland, Dec 1998.

7. D. Raskin, D. Stoneback, "Broadband Return Systems for hybrid fiber/coax cable TV networks.", Reading, Prentice Hall. Publishers, Inc. 1998

8. DOCSIS Acceptance Test Suit, Spirent Communication Inc., Available on Web page: http://www.spirentcom.com

9. J. Guojun, G. Yang, B. R. Crowley, D. Agarwal, "Network Characterization Service". Available on Web page http://www-didc.lbl.gov/NCS

10. N. Chan Hung, B.Carro, R.Mompo, J.Redoli, "Monitoring the Hybrid fiber coaxial on the transport layer". Proceeding of the European Conference on Networks and Optical communications NOC2001, pp249-256, IOS press, UK, July 2001.

11. B. Carro, N. Chan Hung, J. Redoli, R. Mompó, "Link-level effect of a noisy channel over data transmission on the return path of an HFC network", Accepted paper for Globecom 2001, Texas USA.

12. P. Tzerefos, "On the performance and scalability of digital upstream DOCSIS 1.0 conformant CATV channels", Ph.D. Dissertation, University of Sheffield, UK, Oct 1999.

13. R. Ludwig, A. Konrad, A. D. Joseph, "Optimizing the End-to-End performance of reliable flows over wireless links", Proceeding of MobiCom 99.

14. N. Chan Hung, "Systematic study on Hybrid Fiber Coaxial network preventative maintenance and the performance of Internet applications over HFC networks", Ph.D. Dissertation, University of Valladolid, Spain, Jan 2002. Available on http://www.portalvn.com/hungnc/CurrWork.htm 